\title{
The Effect of Phacoemulsification on Intraocular Pressure in Eyes with Hyperfiltration Following Trabeculectomy: A Prospective Study
}

\author{
Ivano Riva · Andreas Katsanos · Francesco Oddone · Luciano Quaranta
}

Received: October 18, 2017 / Published online: December 8, 2017

(c) Springer Healthcare Ltd., part of Springer Nature 2017

\begin{abstract}
Introduction: The aim of this study was to evaluate the effect of visually non-significant cataract extraction in patients with hypotony maculopathy and reduced visual acuity due to over-filtering blebs after trabeculectomy.

Methods: Patients with intraocular pressure (IOP) $<6 \mathrm{mmHg}$ and documented hypotony maculopathy due to over-filtering blebs after trabeculectomy were prospectively recruited. Eligible patients underwent visually non-significant cataract phacoemulsification, no earlier than 12 weeks from the diagnosis of hypotony maculopathy. IOP and visual acuity before and after phacoemulsification were compared at 1 and 3 months from surgery. Correlations between age, time interval between surgeries, baseline IOP, bleb type and IOP and visual
\end{abstract}

Enhanced content To view enhanced content for this article go to http://www.medengine.com/Redeem/ 46FCF060120B888A.

I. Riva $\cdot$ F. Oddone

IRCCS Fondazione GB Bietti per l'Oftalmologia, Rome, Italy

A. Katsanos

Department of Ophthalmology, University of Ioannina, Ioannina, Greece

L. Quaranta $(\square)$

Department of Medical and Surgical Specialties,

University of Brescia, Brescia, Italy

e-mail: luciano.quaranta@unibs.it acuity changes at 3 months after phacoemulsification were investigated.

Results: From January 2010 to September 2014, 20 consecutive adult patients met the inclusion criteria. Before phacoemulsification, mean IOP was $3.1 \pm 1.6 \mathrm{mmHg}$. Following phacoemulsification, mean IOP increased to $8.6 \pm 4.1 \mathrm{mmHg}$ at 1 month $(p<0.01)$ and to $9.1 \pm 4.3 \mathrm{mmHg}$ at 3 months $(p<0.01)$. IOP elevation following phacoemulsification was observed in 16 of 20 eyes (80\%). Mean visual acuity improved from Snellen $0.5 \pm 0.1$ to $0.6 \pm 0.1$ at 1 month $(p<0.01)$ to $0.7 \pm 0.2$ at 3 months $(p<0.01)$ after phacoemulsification. In 4 eyes in which the IOP was not elevated, surgical revision of the previous trabeculectomy was performed. No significant correlations between investigated variables, visual acuity and IOP changes at 3 months after phacoemulsification were found.

Conclusion: Phacoemulsification of visually non-significant cataract appears to be a safe and effective technique for managing chronic ocular hypotony with deep anterior chamber due to over-filtering blebs.

Keywords: Bleb; Glaucoma; Hypotony; Hypotony maculopathy; Trabeculectomy 


\section{INTRODUCTION}

The perioperative use of antimetabolites has been shown to enhance the long-term efficacy of trabeculectomy (TRB) and also increase the incidence of postoperative complications [1-3]. In eyes with very low intraocular pressure (IOP) due to over-filtration or bleb leakage, sight-threatening complications can occur $[3,4]$.

Over-filtration after TRB is not uncommon, and is characterized by diffusely enlarged blebs, ocular discomfort and, in more severe cases, reduction in visual acuity due to hypotony maculopathy. Different therapeutic approaches have been attempted for this condition, including bleb cryotherapy [5], autologous blood injection $[4,6]$, re-suturing of the scleral flap $[7,8]$ and conjunctival compression sutures [9]. The majority of these interventions aims to induce a fibro-vascular reaction at the level of the bleb, thus increasing conjunctival resistance to aqueous outflow and consequently reducing over-filtration.

Cataract surgery has been shown to adversely affect well-functioning TRB blebs [10-12]. Indeed, it has been shown that cataract surgery increases the risk of TRB failure, and this risk is higher if the time between TRB and cataract surgery is relatively short [13]. The mechanism implicated in TRB failure following cataract surgery is thought to involve anterior chamber inflammation that promotes fibrovascular proliferation and subconjunctival healing $[10,14-16]$. Alterations in the consistency of the aqueous humor after intraocular surgery seem to play a role [17], since upregulation of fibrogenic cytokines in the aqueous humor has been documented [18].

As cataract surgery is an inflammation-inducing procedure that promotes sub-conjunctival healing in eyes with previous TRBs, it may be beneficial in patients with over-filtering blebs and hypotony. In the present study, we report the effect of uncomplicated visually non-significant cataract extraction in patients with ocular hypotony due to an over-filtering TRB bleb.

\section{METHODS}

In this prospective interventional case series, patients with visually non-significant cataract and over-filtering blebs causing hypotony, after a TRB with intraoperative use of mitomycin C $(0.3 \mathrm{mg} / \mathrm{ml}$ for $3 \mathrm{~min})$, were included. All procedures followed were in accordance with the ethical standards of the responsible committee on human experimentation (institutional and national) and with the Helsinki Declaration of 1964 , as revised in 2013. Informed consent was obtained from all patients for being included in the study. The trial was not registered on a public repository because the data obtained for the study were in accordance with our current clinical practice.

All patients had their TRB performed at the Glaucoma Service of the University of Brescia, Brescia, Italy. Hypotony was defined as IOP $<6 \mathrm{~mm} \mathrm{Hg}$, with reduced visual acuity due to funduscopic and optical coherence tomography (OCT) documented macular folds. Only patients with deep anterior chamber and over-filtering blebs showing no sign of resolution for at least 12 weeks after uncomplicated TRB were considered for phacoemulsification. Before considering this approach, conservative measures were tried in these patients, such as topical steroids suspension, sub-conjunctival peri-bleb injections of autologous blood, topical aminoglycoside administration (e.g., gentamycin). Patients with a follow-up of at least 3 months after the phacoemulsification were considered for analysis.

All TRBs and cataract surgeries were performed by the same experienced surgeon (LQ). TRBs were performed as proposed by Papadopoulos and Khaw [19], while phacoemulsification was performed under topical anesthesia (lidocaine hydrochloride $4 \%$ eyedrops; Alfa Intes srl, Casoria, Italy) through a $2.2 ; \mathrm{mm}$ temporal clear corneal incision. Foldable acrylic intraocular lenses (AcrySof ${ }^{\circledR}$ SN60AT; Alcon Laboratories, Ft Worth, Tx, USA) were implanted in the capsular bag. The post-operative medical regimen included tobramycin eyedrops (Tobral; Alcon Italia, Milan, Italy) four times daily and flurometholone acetate eyedrops (Flarex 0.1\%; Alcon Italia) twice daily for 
10 days. The small dose of this relatively mild steroid given for a short period of time was decided on because it was deemed appropriate to allow for a certain postoperative inflammation that would conceivably promote bleb healing.

According to our current clinical practice, patients undergoing phacoemulsification were examined before surgery (baseline visit), at 1 week, and then at 1 and 3 months after surgery. Additional visits were performed at the discretion of the surgeon. Clinical examinations at baseline and in all follow-up visits included determination of best-corrected visual acuity (BCVA), Goldmann applanation tonometry, bleb evaluation at the slit lamp, fundoscopy and OCT examination. At baseline, lens opacity was graded using the Lens Opacities Classification System III (LOCS III) [20]. Only eyes classified as having no cataract or non-visually significant cataract (LOCS III score $\leq$ N01/NC1, C1 and P1) were included in the study. To avoid any effect of cataract on BCVA, LOCS III score at baseline visit was compared with the score obtained from patients' charts before TRB, and only eyes with matching data were included. Filtering blebs were clinically categorized at the slit lamp according with their extension on the scleral surface. Type I were defined blebs with an extension $>270^{\circ}$, whereas Type II were defined blebs with an extension $<270^{\circ}$.

Surgical success was defined as IOP $\geq 6 \mathrm{~mm}$ $\mathrm{Hg}$ at the 3-month follow-up visit, without additional medication. If IOP did not increase 3 months after phacoemulsification, surgical revision of TRB site was performed. In this case, the conjunctiva was reopened at the site of the previous trabeculectomy and tight 10.0 nylon sutures were applied to the scleral flap, in a manner that no flow of aqueous humor from the flap was detectable.

A non-parametric Wilcoxon test was used to compare continuous variables, due to the relative small sample size and the skewed distribution of data. Spearman's correlation coefficient was used to evaluate the correlation between continuous variables. The statistical significance was set at 0.05 for a bilateral test. Analyses were carried out using the SAS Software, v.9.2 (SAS Institute, Cary, NC, USA).

\section{RESULTS}

From January 2010 to September 2014, 20 consecutive adult patients met the inclusion criteria and were included in the study. Table 1 shows the demographics and clinical characteristics of the patients. On average (mean $\pm \mathrm{SD}$ ), phacoemulsification was performed $23.9 \pm 7.1$ weeks after TRB. No complications occurred during or immediately after surgery.

At baseline, mean IOP was $3.1 \pm 1.6 \mathrm{~mm} \mathrm{Hg}$. Following phacoemulsification, the mean IOP increased to $8.6 \pm 4.1$ after 1 month $(p<0.01)$ and to $9.1 \pm 4.3 \mathrm{mmHg}$ after 3 months $(p<0.01)$ (Table 2). IOP elevation was observed in the first post-operative month, and was sustained until the 3-month follow-up visit (1month vs. 3 -month IOP, $p=0.15$ ). Surgical success was achieved in 16 of 20 patients (80\%).

Mean visual acuity improved from Snellen $0.5 \pm 0.1$ (range 0.2-0.7) at baseline to $0.6 \pm 0.1$ (range $0.2-0.9, p<0.01$ ) at 1 month and to $0.7 \pm 0.2$ at 3 months (range $0.3-1.0, p<0.01$ ) after phacoemulsification (Table 2 ). In all cases

Table 1 Characteristics of the patients included in the study

\begin{tabular}{ll}
\hline Characteristic & Overall \\
\hline Number of patients/eyes & $20 / 20$ \\
Age, years & $58.3(7.5)$ \\
Mean (SD) & $44-71$ \\
Min-max & \\
Sex, $n$ (\%) & $11(55.0)$ \\
Men & $9(45.0)$ \\
Women & $0.64(0.29)$ \\
Lens opacity grading (LOCS III score), mean $(\mathrm{SD})$ \\
Nuclear color/opalescence (N/NC) & $0.72(0.33)$ \\
Cortical (C) & $0.27(0.33)$ \\
Posterior subcapsular (P) & \\
Timing of phacoemulsification, weeks & $23.9(7.1)$ \\
Mean (SD) & $14-36$ \\
Min-max
\end{tabular}


Table 2 Intraocular pressure and visual acuity before and after surgery

\begin{tabular}{|c|c|c|c|c|c|}
\hline & Baseline & 1 month & 3 month & $\begin{array}{l}\text { Mean difference 1-month } \\
(95 \% \mathrm{CI})\end{array}$ & $\begin{array}{l}\text { Mean difference 3-month } \\
\text { (95\% CI) }\end{array}$ \\
\hline \multicolumn{6}{|l|}{ IOP (mmHg) } \\
\hline Mean (SD) & $3.1(1.6)$ & $8.6(4.1)$ & $9.1(4.3)$ & $5.5(3.8-7.2)$ & $6.0(4.2-7.7)$ \\
\hline Min-max & $0-6$ & $2-17$ & $2-16$ & $p<0.01$ & $p<0.01$ \\
\hline \multicolumn{6}{|c|}{ BCVA, Snellen } \\
\hline Mean (SD) & $0.5(0.1)$ & $0.6(0.1)$ & $0.7(0.2)$ & $0.1(0.05-0.2)$ & $0.2(0.1-0.3)$ \\
\hline Min-max & $0.2-0.7$ & $0.2-0.9$ & $0.3-1.0$ & $p<0.01$ & $p<0.01$ \\
\hline
\end{tabular}

$I O P$ intraocular pressure, $B C V A$ best corrected visual acuity expressed in Snellen's decimal fraction
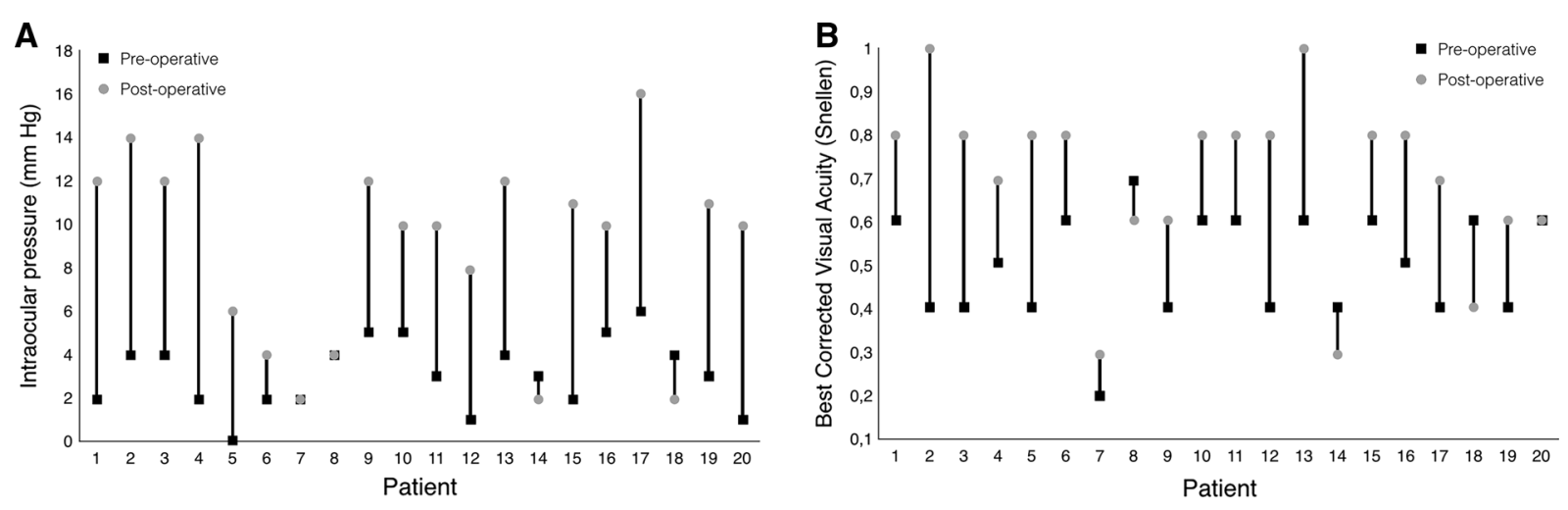

Fig. 1 Intraocular pressure and best corrected visual acuity change at 3-month follow-up for each studied patient; $\mathbf{a}$ intraocular pressure modification; $\mathbf{b}$ best corrected visual acuity modification

except one in which surgical success was achieved, visual acuity improved and macular folds either became less prominent or disappeared at OCT examination (Fig. 1).

At baseline, Type I and Type II blebs were observed in 15 (75\%) and 5 (25\%) eyes, respectively. At 3-month follow-up visit, Type I and Type II blebs were observed in 9 (45\%) and 11 (55\%) eyes, respectively. This difference in bleb morphology before and after phacoemulsification was statistically significant $(p<0.01)$. IOP and visual acuity changes at 3 months after cataract surgery did not correlate with patient age $(p=0.29$ and $p=0.94)$, time interval between filtering surgery and phacoemulsification $(p=0.34$ and $p=0.4)$, baseline IOP $(p=0.7$ and $p=0.73)$ and bleb type $(p=0.73$ and $p=0.66)$.

In four eyes (20\%), the IOP did not increase when compared with the value before phacoemulsification. In these cases, surgical revision of the scleral flap was performed. IOP and visual acuity of these four eyes 1 month after surgery are shown in Table 3. In two eyes (50\%), medical therapy was needed because of inadequate IOP control.

\section{DISCUSSION}

The introduction of antimetabolites as an adjunct to TRB has increased the success of the procedure, but also the risk of vision-threatening complications, such as long-lasting hypotony [4, 21-28]. Cataract is common in eyes that have undergone TRB. Indeed, the Advanced Glaucoma Intervention Study has shown that TRB may increase the risk of cataract formation by $78 \%$ [29]. However, the patients included in our study did not have clinically significant lens 
Table 3 Intraocular pressure and best corrected visual acuity in patients undergone surgical revision of trabeculectomy

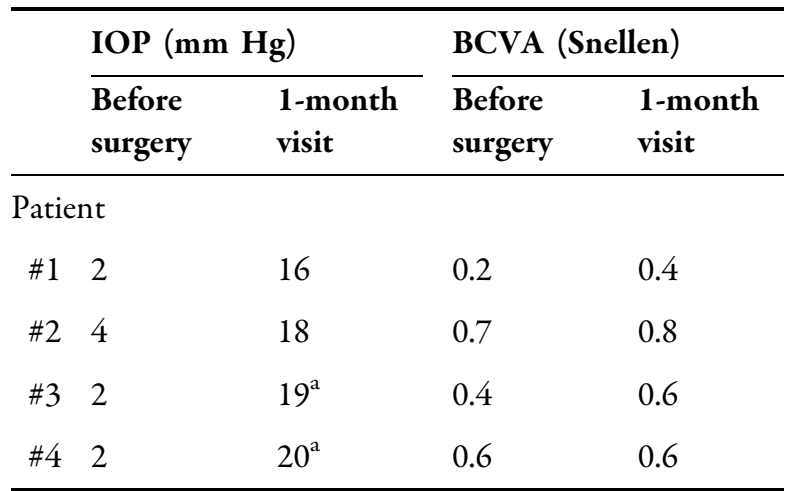

IOP intraocular pressure, $B C V A$ best corrected visual acuity expressed in Snellen's decimal fraction

a Patients needed topical hypotensive therapy

opacity, at least when examined ophtalmoscopically. Consequently, in our patients with chronic hypotony maculopathy, the reduced preoperative visual acuity can reasonably be assumed to be due to their macular condition, rather than due to cataract. Thus, we believe that the improvement in visual acuity after phacoemulsification represents the effect of IOP elevation on macular structure and function, rather than the effect of cataract lens extraction.

Excessive scarring at the conjunctival-scleral interface or within the scleral flap bed is the main reason for TRB failure [6]. There are several factors associated with excessive conjunctival healing and scarring, such as race [30], previous use of topical medication [31], and age [32]. The increased bioavailability of inflammatory mediators after cataract surgery can also increase the risk of TRB bleb failure. Using laser flare photometry, Chee et al. found that anterior chamber flare is increased after phacoemulsification, while approximately 30 days are needed before flare levels return to normal [33]. Using the same technique, Siriwardena et al. compared anterior chamber flare in 131 patients who underwent TRB and in 148 patients who underwent phacoemulsification [34]. The authors found significantly higher levels of anterior chamber flare in the phacoemulsification group at all postoperative time points up to 3 months. Anterior chamber flare in the phacoemulsification group returned to preoperative levels by month 6 . In contrast, in the TRB group, flare levels returned to normal after 4 weeks. The authors assumed that the prolonged low-grade inflammation associated with phacoemulsification is likely due to lens crystallines, the effect of ultrasound energy, and the high volume of fluid circulating through the eye during surgery. More recently, Husain et al. demonstrated in a randomized clinical trial that cataract surgery after TRB increases the risk of TRB failure, and that this risk is higher if the time between TRB and cataract surgery is shorter [13]. Conceivably, the elevated concentration of inflammatory mediators in the aqueous humor following phacoemulsification represents a risk factor for bleb failure.

In our series, phacoemulsification with reduced topical steroid therapy in the post-operative period increased the IOP in a significant percentage of eyes $(80 \%)$ with chronic hypotony due to an over-filtrating TRB. Moreover, bleb extension at 3 months after phacoemulsification showed a significant reduction in six eyes. Our results are in accordance with those reported by Doyle et al. in a smaller sample of patients (nine eyes) [35]. A potentially relevant difference between our technique and that of Doyle et al. is that these authors were leaving viscoelastic in the anterior chamber at the end of the procedure [35]. It is unclear if the passage of viscoelastic material in the subconjunctival space could further enlarge the filtration area. In addition, it could be argued that viscoelastic material remaining under the bleb could potentially reduce the healing effect promoted by phacoemulsification-induced inflammation. Moreover, in the series of Doyle et al. [35], one patient had a transient but large IOP spike. In our series, no IOP spikes were recorded in any patient.

Patients included in this study had prolonged over-filtering conjunctival blebs associated with hypotony, and did not respond to conservative measures. Therefore, we believe that spontaneous resolution of hypotony would be unlikely in these patients, and that our results are explained by our intervention, rather than the natural course of the condition. Four patients in our series did not show any IOP 
increase after phacoemulsification. In these patients, surgical revision of the scleral flap was performed. However, two eyes (50\%) developed high IOP, and topical hypotensive medical therapy was needed to control IOP. These data are in accordance with the literature [7], and make flap revision a suboptimal way to manage these patients.

Hypotony caused by excessive bleb filtration often resolves with conservative postoperative management [36]. In our clinical practice, if an anterior chamber is present and there is no sign of hypotony maculopathy, a wait-and-see behavior is generally adopted, tapering topical steroids until IOP spontaneously rises. However, when hypotony maculopathy develops, prompt intervention is needed. Conservative procedures are the first step. Sub-conjunctival peribleb injection of autologous blood, associated with topical aminoglycoside administration (e.g., gentamycin), is our first intervention. If no rise in IOP is evidenced shortly, a more invasive procedure is chosen. In patients with over-filtrating blebs extending less than $270^{\circ}$ on the scleral surface, two or more 9.0 nylon compression sutures are placed. In our hands, this procedure resolved hypotony in $64.4 \%$ of eyes in a series of patients affected with hypotony maculopathy and over-filtration [9]. If bleb extends over $360^{\circ}$, or compression sutures are not effective, surgical revision of the scleral flap is suggested. This procedure is generally effective; however, IOP spikes after surgery are not rare, and the risk of intra-operative complications is high [7]. In comparison with these procedures, phacoemulsification of clear lens has shown excellent results in this study, resolving hypotony in $80 \%$ of patients at 3 months, with no intra- or extra-operative complications.

Long-term results of interventions for overfiltering blebs associated with hypotony maculopathy is unknown. In a small case series of eyes affected with hypotony maculopathy after trabeculectomy by Schwartz et al., four eyes underwent resuturing of the scleral flap to reverse excessive filtration. The procedure resulted in improved visual acuity in all cases, with IOP between 6 and $17 \mathrm{~mm} \mathrm{Hg}$ with no topical medications (minimum follow-up:
18 months) [37]. Trans-conjunctival suturing of the scleral flap has been proposed by Eha et al. to treat eyes with hypotony maculopathy attributable to over-filtration after trabeculectomy with MMC. This procedure was performed in 53 eyes with a mean baseline IOP of $3.55 \mathrm{mmHg}( \pm 2.05$; range $0-8 \mathrm{mmHg})$ and a mean visual acuity of 0.8 LogMar. At 4 -year follow-up, IOP and visual acuity increased to $9.5 \mathrm{mmHg}$ and 0.3 LogMar, respectively. Macular folds resolved in all patients [38]. Okada et al. performed peri-bleb injections of autologous blood in five eyes with excess filtration and hypotony after trabeculectomy, but without hypotony maculopathy. After a mean 31-month follow-up, all eyes had well-controlled IOP while visual acuity in three eyes was much improved [39]. Due to small sample size and no rigorous methodology, further research is needed to establish the best surgical approach for these patients and the long-term outcomes.

Hypotony can induce a shortening of the eye, which may affect biometry. If the eye is restored to its pre-TRB axial length, the IOL might not be of the appropriate power. For this reason, it is important to always assess ocular biometry before TRB, in order to also implant the correct IOL in these eyes affected with hypotony.

In our study, IOP and visual acuity changes after 3-month follow-up were not correlated with age, time interval between surgeries, baseline IOP and bleb type. However, these results should be interpreted with caution, due to the small sample size of the study, the relatively short follow-up and the absence of a control group. Another limitation of our study was the absence of an objective grading for macular folds. However, OCT has demonstrated to be extremely sensitive in detection of macular folds in patients affected with hypotony maculopathy [40], and subjective comparison of OCT scans may be an accurate approach to follow-up this condition.

\section{CONCLUSION}

In conclusion, phacoemulsification of visually non-significant cataract appears to be a safe and effective technique for managing chronic ocular 
hypotony with deep anterior chamber due to over-filtering TRB blebs.

\section{ACKNOWLEDGEMENTS}

Funding. The contribution of the Fondazione Bietti in this study was supported by Ministry of Health and Fondazione Roma, Italy. No funding was received for the publication of this article.

Authorship. All named authors meet the International Committee of Medical Journal Editors (ICMJE) criteria for authorship for this manuscript, take responsibility for the integrity of the work as a whole, and have given final approval to the version to be published.

Disclosures. Ivano Riva, Andreas Katsanos, Francesco Oddone and Luciano Quaranta have nothing to disclose.

Compliance with Ethics Guidelines. All procedures followed were in accordance with the ethical standards of the responsible committee on human experimentation (institutional and national) and with the Helsinki Declaration of 1964, as revised in 2013. Informed consent was obtained from all patients for being included in the study.

Data Availability. The datasets during and/ or analyzed during the current study are available from the corresponding author on reasonable request.

\section{REFERENCES}

1. Wilkins $\mathrm{M}$, Indar $\mathrm{A}$, Wormald R. Intra-operative mitomycin C for glaucoma surgery. Cochrane Database Syst Rev. 2005;19(4):CD002897.

2. Jampel HD, Musch DC, Gillespie BW, et al. Perioperative complications of trabeculectomy in the collaborative initial glaucoma treatment study (CIGTS). Am J Ophthalmol. 2005;140:16-22.

3. The Fluorouracil Filtering Surgery Study Group. Five-year follow-up of the Fluorouracil Filtering Surgery Study. Am J Ophthalmol. 1996;121:349-66.
4. Ticho U, Ophir A. Late complications after glaucoma filtering surgery with adjunctive 5-fluorouracil. Am J Ophthalmol. 1993;115:506-10.

5. El-Harazi SM, Fellman RL, Feldman RM, Dang YN, Chuang AZ. Bleb window cryopexy for the management of oversized, misplaced blebs. J Glaucoma. 2001;10:47-50.

6. Jampel HD, Solus JF, Tracey PA, et al. Outcomes and bleb-related complications of trabeculectomy. Ophthalmology. 2012;119:712-22.

7. Bitrian E, Song BJ, Caprioli J. Bleb revision for resolution of hypotony maculopathy following primary trabeculectomy. Am J Ophthalmol. 2014;158(597-604):e591.

8. Eha J, Hoffmann EM, Wahl J, Pfeiffer N. Flap suture-a simple technique for the revision of hypotony maculopathy following trabeculectomy with mitomycin C. Graefes Arch Clin Exp Ophthalmol. 2008;246:869-74.

9. Quaranta L, Riva I, Floriani IC. Outcomes of conjunctival compression sutures for hypotony after glaucoma filtering surgery. Eur J Ophthalmol. 2013;23:593-6.

10. Rebolleda G, Munoz-Negrete FJ. Phacoemulsification in eyes with functioning filtering blebs: a prospective study. Ophthalmology. 2002; 109:2248-55

11. Casson R, Rahman R, Salmon JF. Phacoemulsification with intraocular lens implantation after trabeculectomy. J Glaucoma. 2002;11:429-33.

12. Mietz H, Andresen A, Welsandt G, Krieglstein GK. Effect of cataract surgery on intraocular pressure in eyes with previous trabeculectomy. Graefes Arch Clin Exp Ophthalmol. 2001;239:763-9.

13. Husain R, Liang S, Foster PJ, et al. Cataract surgery after trabeculectomy: the effect on trabeculectomy function. Arch Ophthalmol. 2012;130:165-70.

14. Sharma TK, Arora S, Corridan PG. Phacoemulsification in patients with previous trabeculectomy: role of 5-fluorouracil. Eye (Lond). 2007;21:780-3.

15. Addicks EM, Quigley HA, Green WR, Robin AL. Histologic characteristics of filtering blebs in glaucomatous eyes. Arch Ophthalmol. 1983;101: 795-8.

16. Khaw PT, Chang L, Wong TT, et al. Modulation of wound healing after glaucoma surgery. Curr Opin Ophthalmol. 2001;12:143-8.

17. Joseph JP, Grierson I, Hitchings RA. Chemotactic activity of aqueous humor. A cause of failure of 
trabeculectomies? Arch Ophthalmol. 1989;107:69-74.

18. Allen JB, Davidson MG, Nasisse MP, Fleisher LN, McGahan MC. The lens influences aqueous humor levels of transforming growth factor-beta 2 . Graefes Arch Clin Exp Ophthalmol. 1998;236:305-11.

19. Papadopoulos $M$, Khaw PT. Improving glaucoma filtering surgery. Eye (Lond). 2001;15:131-2.

20. Chylack LT Jr, Wolfe JK, Singer DM, et al. The lens opacities classification system III. The Longitudinal Study of Cataract Study Group. Arch Ophthalmol. 1993;111:831-6.

21. Greenfield DS, Liebmann JM, Jee J, Ritch R. Lateonset bleb leaks after glaucoma filtering surgery. Arch Ophthalmol. 1998;116:443-7.

22. Greenfield DS, Suner IJ, Miller MP, et al. Endophthalmitis after filtering surgery with mitomycin. Arch Ophthalmol. 1996;114:943-9.

23. Soltau JB, Rothman RF, Budenz DL, et al. Risk factors for glaucoma filtering bleb infections. Arch Ophthalmol. 2000;118:338-42.

24. Budenz DL, Chen PP, Weaver YK. Conjunctival advancement for late-onset filtering bleb leaks: indications and outcomes. Arch Ophthalmol. 1999;117:1014-9.

25. Waheed S, Ritterband DC, Greenfield DS, et al. Bleb-related ocular infection in children after trabeculectomy with mitomycin C. Ophthalmology. 1997;104:2117-20.

26. Stamper RL, McMenemy MG, Lieberman MF. Hypotonous maculopathy after trabeculectomy with subconjunctival 5-fluorouracil. Am J Ophthalmol. 1992;114:544-53.

27. Costa VP, Wilson RP, Moster MR, Schmidt CM, Gandham S. Hypotony maculopathy following the use of topical mitomycin C in glaucoma filtration surgery. Ophthalmic Surg. 1993;24:389-94.

28. Zacharia PT, Deppermann SR, Schuman JS. Ocular hypotony after trabeculectomy with mitomycin C. Am J Ophthalmol. 1993;116:314-26.
29. Investigators A. The Advanced Glaucoma Intervention Study: 8. Risk of cataract formation after trabeculectomy. Arch Ophthalmol. 2001;119:1771-9.

30. Husain R, Clarke JC, Seah SK, Khaw PT. A review of trabeculectomy in East Asian people-the influence of race. Eye (Lond). 2005;19:243-52.

31. Boimer C, Birt CM. Preservative exposure and surgical outcomes in glaucoma patients: the PESO study. J Glaucoma. 2013;22:730-5.

32. Broadway DC, Chang LP. Trabeculectomy, risk factors for failure and the preoperative state of the conjunctiva. J Glaucoma. 2001;10:237-49.

33. Chee SP, Ti SE, Sivakumar M, Tan DT. Postoperative inflammation: extracapsular cataract extraction versus phacoemulsification. J Cataract Refract Surg. 1999;25:1280-5.

34. Siriwardena D, Kotecha A, Minassian D, Dart JK, Khaw PT. Anterior chamber flare after trabeculectomy and after phacoemulsification. Br J Ophthalmol. 2000;84:1056-7.

35. Doyle JW, Smith MF. Effect of phacoemulsification surgery on hypotony following trabeculectomy surgery. Arch Ophthalmol. 2000;118:763-5.

36. Azuara-Blanco A, Katz LJ. Dysfunctional filtering blebs. Surv Ophthalmol. 1998;43:93-126.

37. Schwartz GF, Robin AL, Wilson RP, et al. Resuturing the scleral flap leads to resolution of hypotony maculopathy. J Glaucoma. 1996;5:246-51.

38. Eha J, Hoffmann EM, Pfeiffer N. Long-term results after transconjunctival resuturing of the scleral flap in hypotony following trabeculectomy. Am J Ophthalmol. 2013;155:864-9.

39. Okada $\mathrm{K}$, Tsukamoto $\mathrm{H}$, Masumoto $\mathrm{M}$, et al. Autologous blood injection for marked overfiltration early after trabeculectomy with mitomycin C. Acta Ophthalmol Scand. 2001;79:305-8.

40. Costa VP, Arcieri ES. Hypotony maculopathy. Acta Ophthalmol Scand. 2007;85:586-97. 